TU-921

\title{
A Gravitino-rich Universe
}

\author{
Kwang Sik Jeong*\| and Fuminobu Takahashi" \\ Department of Physics, Tohoku University, Sendai 980-8578, Japan
}

\begin{abstract}
The gravitino may well play an important role in cosmology, not only because its interactions are Planck-suppressed and therefore long-lived, but also because it is copiously produced via various processes such as particle scatterings in thermal plasma, and (pseudo) modulus and inflaton decays. We study a possibility that the early Universe was gravitino-rich from various aspects. In particular, a viable cosmology is possible, if high-scale supersymmetry is realized in nature as suggested by the recent discovery of the standard-model like Higgs boson of mass about $125-126 \mathrm{GeV}$. We find that the Universe can be even gravitino-dominated, in which case there will be an entropy dilution by the gravitino decay. If the gravitino abundance is proportional to the reheating temperature, both the maximal baryon asymmetry in leptogenesis and the dark matter from the gravitino decay become independent of the reheating temperature. The dark matter candidate is the Wino-like neutralino, whose mass is suppressed compared to the anomaly-mediation relation.
\end{abstract}

* email: ksjeong@tuhep.phys.tohoku.ac.jp

$\dagger$ email: fumi@tuhep.phys.tohoku.ac.jp 


\section{Introduction}

The recent discovery of the standard-model (SM) like Higgs boson of mass about 125 $126 \mathrm{GeV}$ [1, 2] may imply high-scale supersymmetry (SUSY) [3, 4]. SUSY relates fermions to bosons and vice versa, and adds the superpartner of each particle in nature; hence, in a supersymmetric theory there will be a fermion that partners with the graviton, called the gravitino, which becomes massive via the super-Higgs mechanism after SUSY gets spontaneously broken. The interactions of the gravitino are suppressed by either the Planck scale or the SUSY breaking scale, and therefore it is naturally long-lived.

The gravitinos are produced in various processes. In the early Universe, the gravitinos are produced by particle scatterings in thermal plasma [5, 6, 7]. Also, it is known that any scalar field with a non-vanishing vacuum expectation value (VEV) generically decays into gravitinos, if kinematically allowed. In particular, a large amount of gravitinos can be produced by the (pseudo) modulus [8, 9, 10] and inflaton [11, 12, 13, 14, 15] decays.

If the gravitinos are abundant in the early Universe, they may play an important role in cosmology, because of their longevity. Indeed, it is well-known that too many gravitinos significantly affect the evolution of the Universe in contradiction with observations; the cosmological gravitino problem [16, 17, 18]. For instance, if the gravitino mass is around the weak scale, it typically decays during big bang nucleosynthesis (BBN), altering the light element abundances in contradiction with observations. In the case of high-scale SUSY, however, the gravitino can decay before BBN, and the success of the standard BBN remains intact. Thus, the high-scale SUSY may lead to a viable cosmology even if many gravitinos are produced in the early Universe. The main purpose of this paper is to study such gravitino-rich Universe from both cosmological and phenomenological aspects.

What is peculiar to the gravitino is that it is coupled to any sectors in nature. Thus, once produced, the gravitinos will distribute their energy to all the lighter particles including the SM particles. For instance, the gravitino decay produces the lightest ordinary supersymmetric particle. If it is the lightest supersymmetric particle (LSP) and if the R-parity is conserved, the LSPs thus produced may account for the observed dark matter (DM) abundance. Alternatively, if there are light degrees of freedom in a hidden sector, the gravitino necessarily decays into them. Some of the hidden-sector particles thus 
produced may contribute to DM, if they are long-lived and non-relativistic. If they are relativistic at the $\mathrm{BBN}$ and recombination epoch, they will increase the expansion rate, which may account for the dark radiation hinted by recent observations [22, 23]. (See e.g. Refs. [24, 25, 26, 27, 28, 29, 30, 31] for the models.) Also, if the inflation took place in a hidden sector, the only way to reheat the visible sector may be through the gravitino production; the gravitino may play a role of messenger because of its universal couplings. Thus, the gravitino-rich Universe seems to have various interesting implications.

In this paper we shall study if the Universe becomes gravitino-rich by considering several production processes of the gravitinos; thermal production as well as non-thermal production by the (pseudo) modulus and inflaton decays. We find that the gravitinorich Universe is realized for a wide range of parameters, and the Universe even becomes gravitino-dominated in some cases. In the latter case, there is an entropy dilution by the gravitino decay. Interestingly, the gravitino abundance and therefore its dilution factor are proportional to the reheating temperature in the case of thermal production and the nonthermal production by the pseudo-modulus decay. Then, the maximal baryon asymmetry in thermal leptogenesis becomes independent of the reheating temperature. Assuming the Wino-like LSP of mass several hundred GeV, the LSP abundance can account for the observed DM. For this, the anomaly mediation relation for the gaugino masses must be modified, requiring a mild cancellation of order $10 \%$ in the Wino mass. Note that both the baryon asymmetry and the neutralino LSP abundance are independent of the reheating temperature in this case.

The rest of this paper is organized as follows. In Sec. 2 we briefly discuss the gravitino lifetime and abundance. We study various production processes of the gravitinos in Sec. 3 , In Sec. 4 we discuss cosmological aspects of the gravitino-rich Universe, and study the maximal baryon asymmetry in the thermal leptogenesis as well as the DM abundance. In Sec. 5 we discuss how the gaugino mass relation can be deviated from the anomaly mediation relation. The last section is devoted for discussion and conclusions. 


\section{Gravitino abundance}

Let us summarize here the abundance and lifetime of the gravitino. For the moment we assume the minimal particle content, namely, the minimal supersymmetric standard model (MSSM) particles and the gravitino. Later we shall consider a case that there are light degrees of freedom in the hidden sector.

The decay rate of the gravitino into the MSSM particles is approximately given by

$$
\Gamma_{3 / 2}\left(\psi_{3 / 2} \rightarrow \mathrm{MSSM}\right) \simeq \frac{193}{384 \pi} \frac{m_{3 / 2}^{3}}{M_{P}^{2}},
$$

where $m_{3 / 2}$ is the gravitino mass, $M_{P} \simeq 2.4 \times 10^{18} \mathrm{GeV}$ is the reduced Planck mass, and we have assumed that the gravitino is heavier than all the MSSM particles, for simplicity. The decay temperature of the gravitino, $T_{3 / 2}$, is defined by

$$
\begin{aligned}
T_{3 / 2} & \equiv\left(\frac{\pi^{2} g_{*}\left(T_{3 / 2}\right)}{90}\right)^{-\frac{1}{4}} \sqrt{\Gamma_{3 / 2} M_{P}} \\
& \simeq 0.15 \mathrm{GeV}\left(\frac{g_{*}\left(T_{3 / 2}\right)}{80}\right)^{-\frac{1}{4}}\left(\frac{m_{3 / 2}}{10^{3} \mathrm{TeV}}\right)^{\frac{3}{2}}
\end{aligned}
$$

where $g_{*}(T)$ counts the relativistic degrees of freedom at temperature $T$. If the gravitino is heavier than several tens $\mathrm{TeV}$, its decay temperature is above a few $\mathrm{MeV}$, avoiding the tight BBN constraint [19]. We adopt $m_{3 / 2}=10^{3} \mathrm{TeV}$ as a reference value in the following.

Let us quantify the gravitino abundance in terms of the ratio of the gravitino number density to the entropy density;

$$
Y_{3 / 2} \equiv \frac{n_{3 / 2}}{s_{i}}
$$

where $s_{i}$ represents the entropy density originated from the inflaton decay. Note that $s_{i}$ does not include the entropy produced by the gravitino decay.

If the gravitinos are abundant, they may come to dominate the energy density of the Universe, since the gravitino is massive and long-lived. The gravitino-dominated Universe is realized if

$$
Y_{3 / 2}>\frac{3}{4} \frac{T_{3 / 2}}{m_{3 / 2}}
$$


This is equivalent to the condition that the entropy produced by the gravitino, $s_{3 / 2}$, be larger than the pre-existing entropy, $s_{i}$. Using Eq. (3), this condition can be rewritten as

$$
Y_{3 / 2} \gtrsim 1.5 \times 10^{-7}\left(\frac{g_{*}\left(T_{3 / 2}\right)}{80}\right)^{-\frac{1}{4}}\left(\frac{m_{3 / 2}}{10^{3} \mathrm{TeV}}\right)^{\frac{1}{2}}
$$

If this condition is met, the entropy dilution factor $\Delta$ is given by

$$
\begin{aligned}
\Delta \equiv \frac{s_{3 / 2}+s_{i}}{s_{i}} & \simeq \frac{n_{3 / 2}}{s_{i}}\left(\frac{n_{3 / 2}}{s_{3 / 2}}\right)^{-1} \\
& \simeq 90\left(\frac{g_{*}\left(T_{3 / 2}\right)}{80}\right)^{\frac{1}{4}}\left(\frac{m_{3 / 2}}{10^{3} \mathrm{TeV}}\right)^{-\frac{1}{2}}\left(\frac{Y_{3 / 2}}{10^{-5}}\right)
\end{aligned}
$$

where $s_{3 / 2}$ represents the entropy density produced by the gravitino decay, and we have used $\left(n_{3 / 2} / s_{3 / 2}\right)=3 T_{3 / 2} / 4 m_{3 / 2}$. Noting that $Y_{3 / 2} \sim 10^{-2}$ when gravitinos are in thermal equilibrium, one can see that the dilution factor varies from 1 to $\sim 10^{5}$ for the gravitino mass around $10^{3} \mathrm{TeV}$.

It is possible that a modulus field dominates the Universe after the reheating, and decays into gravitinos. If the branching fraction of the modulus decay into gravitinos is large enough, both the modulus and gravitino decays produce entropy. In this case the total entropy dilution factor is the same as Eq. (8), and it can be also expressed in terms of the modulus abundance as

$$
\begin{aligned}
\Delta & \simeq \frac{\rho_{X}}{s_{i}} \frac{n_{3 / 2}}{\rho_{X}}\left(\frac{n_{3 / 2}}{s_{3 / 2}}\right)^{-1} \\
& \simeq \frac{4}{3} B_{3 / 2} N_{3 / 2} \frac{m_{3 / 2}}{m_{X} T_{3 / 2}}\left(\frac{\rho_{X}}{s_{i}}\right),
\end{aligned}
$$

where $B_{3 / 2}$ denotes the branching fraction into gravitinos, $N_{3 / 2}$ is the average number of gravitinos for the decay modes into gravitinos, $m_{X}$ and $\rho_{X}$ are the mass and energy density of the modulus $X$. When the modulus mainly decays into a pair of gravitinos, we expect $B_{3 / 2} \simeq 1$ and $N_{3 / 2}=2$.

If ([6]) is not satisfied, the gravitinos do not dominate the energy density of the Universe, but they can still have a significant impact on cosmology. For instance, if its abundance is greater than $\sim 10^{-12}$, the LSPs produced by the gravitino decay tend to overclose the Universe, as long as the annihilation of the LSPs is not effective and the LSP mass is larger 
than or comparable to $\mathcal{O}(100) \mathrm{GeV}$. We therefore adopt the criterion for the gravitino-rich Universe as,

$$
\text { Gravitino-rich Universe : } Y_{3 / 2}>10^{-12}
$$

Of course, there are various possibilities, for instance, the R-parity may be violated, and the QCD axion may be the dominant component of DM, or there may be other lighter SUSY particles in the hidden sector, and so on. A different criterion should be adopted depending on the scenarios of interest. To be concrete, however, we adopt the above criterion in this paper.

\section{$3 \quad$ Production processes}

Now we consider various processes for the gravitino production.

\subsection{Thermal production}

Gravitinos are produced by particle scatterings in thermal plasma, and its abundance is given by [5, 6, 7 ]

$$
Y_{3 / 2}^{(\mathrm{TP})} \simeq 2 \times 10^{-12}\left(\frac{T_{R}}{10^{10} \mathrm{GeV}}\right),
$$

where we have omitted the logarithmic dependence on $T_{R}$ as well as terms that depend on the gaugino masses. The definition of $T_{R}$ is given by

$$
T_{R} \equiv\left(\frac{\pi^{2} g_{*}\left(T_{R}\right)}{90}\right)^{-\frac{1}{4}} \sqrt{\Gamma_{\phi} M_{P}}
$$

where $\Gamma_{\phi}$ denotes the total decay rate of the inflaton, and we assume that the inflaton mainly decays into the MSSM sector. Thus, the gravitino abundance increases in proportion to the reheating temperature.

The gravitino-rich Universe is realized if the reheating temperature satisfies

$$
T_{R} \gtrsim 10^{10} \mathrm{GeV}
$$

while the gravitino dominates the Universe if the reheating temperature is

$$
T_{R} \gtrsim 10^{15} \mathrm{GeV}\left(\frac{g_{*}\left(T_{R}\right)}{80}\right)^{-\frac{1}{4}}\left(\frac{m_{3 / 2}}{10^{3} \mathrm{TeV}}\right)^{\frac{1}{2}}
$$


Thus, an extremely high reheating temperature is required for the gravitino to dominate the Universe.

Note however that the expression for the gravitino abundance (12) does not take account of the longitudinal mode. The contribution from the longitudinal mode, namely the goldstino, becomes relevant if the gauginos are heavier than the gravitino. Furthermore, if the inflaton decays into the SUSY breaking sector 11 the would-be goldstino may be ther-

malized, leading to $Y_{3 / 2}^{(\mathrm{TP})} \sim 10^{-2}$, where we have assumed that the relativistic degrees of freedom is of order $10^{2}$. Thus, it should be kept in mind that the above estimate on the gravitino abundance (12) depends on the assumption about the reheating process and the mass spectrum.

\subsection{Non-thermal production}

Now let us consider non-thermal production of gravitinos. In the following we consider a pseudo modulus in the SUSY breaking sector, a general modulus field, and an inflaton as possible source of gravitinos.

\subsubsection{Pseudo modulus field in the SUSY breaking sector}

We first consider a pseudo-modulus decay into gravitinos. A pseudo modulus in the SUSY breaking sector is a plausible candidate for the gravitino production because of the following reasons; (i) the existence of the pseudo modulus is generic in the SUSY breaking models; (ii) the coherent oscillations are produced because the potential minimum for the pseudo modulus during inflation is generically deviated from the low-energy minimum; (iii) it predominantly decays into a pair of gravitinos.

We study the low-energy effective theory of O'Raifeartaigh type SUSY breaking model. After integrating out the massive fields, the Kähler and super-potentials are

$$
\begin{aligned}
K & =|z|^{2}-\frac{|z|^{4}}{\Lambda^{2}}+\cdots, \\
W & =\mu^{2} z+W_{0},
\end{aligned}
$$

where $z$ is a pseudo modulus field, $\Lambda$ is a cut-off scale, $\mu$ represents the SUSY breaking scale, and the constant $W_{0} \simeq m_{3 / 2} M_{P}^{2}$ is fixed so that the cosmological constant almost

\footnotetext{
${ }^{1}$ The inflaton itself may be a part of the SUSY breaking sector.
} 
vanishes at present. We can assign an R-charge 2 on $z$, which is explicitly broken by $W_{0}$ down to $Z_{2 R}$. All the parameters are set to be real by an appropriate redefinition of $z$ and the $\mathrm{U}(1)_{R}$ transformation.

The $z$ is stabilized at the potential minimum,

$$
\langle z\rangle=\frac{2 \sqrt{3} m_{3 / 2}^{2} M_{P}}{m_{z}^{2}},
$$

where $m_{z}$ is the mass of $z$ given by

$$
m_{z}=\frac{2 \mu^{2}}{\Lambda} .
$$

The F-term of $z$ is given by $F_{z} \simeq-\mu^{2} \simeq \sqrt{3} m_{3 / 2} M_{P}$, and SUSY is indeed broken. The precise value of $\Lambda$ depends on details of the SUSY breaking. We simply assume here that $\Lambda$ is much smaller than the Planck scale so that $z$ is heavier than the gravitino.

Suppose that the $\mathrm{U}(1)_{R}$ symmetry remains a good symmetry during inflation and that the inflation scale, $H_{\mathrm{inf}}$, is larger than $m_{z}$. Then, if $z$ acquires a positive Hubbleinduced mass, $z$ is stabilized near the origin during inflation, and starts to oscillate with an amplitude of $\langle z\rangle$ when $H \sim m_{z}$ after inflation.2 Thus the pseudo-modulus abundance is given by

$$
\frac{\rho_{z}}{s_{i}} \simeq 3 T_{R}\left(\frac{m_{3 / 2}}{m_{z}}\right)^{4},
$$

where we have assumed that the pseudo modulus starts to oscillate before the reheating. On the other hand, if the Hubble parameter during inflation is smaller than $m_{z}$, the pseudo-modulus abundance gets suppressed. If the inflaton at the potential minimum is heavier than $m_{z}$, the pseudo modulus cannot follow the change of the potential minimum, and the coherent oscillations are induced [32, 33]. The abundance is given by

$$
\frac{\rho_{z}}{s_{i}} \simeq 3 T_{R}\left(\frac{m_{3 / 2}}{m_{z}}\right)^{4}\left(\frac{H_{\mathrm{inf}}}{m_{z}}\right)^{4} .
$$

If the inflaton mass at the potential minimum is lighter than $m_{z}$, no coherent oscillations are induced. In the following analysis we use the pseudo-modulus abundance (20). As one can see from Fig. 1, this is the case for the inflation scale larger than $10^{9} \sim 10^{10} \mathrm{GeV}$, which covers many inflation models.

\footnotetext{
${ }^{2}$ Even if the Hubble parameter during inflation exceeds the dynamical scale, it is possible that $z$ is stabilized near the origin, when the Hubble parameter becomes so small after inflation that the low-energy description (16) and (17) are valid.
} 


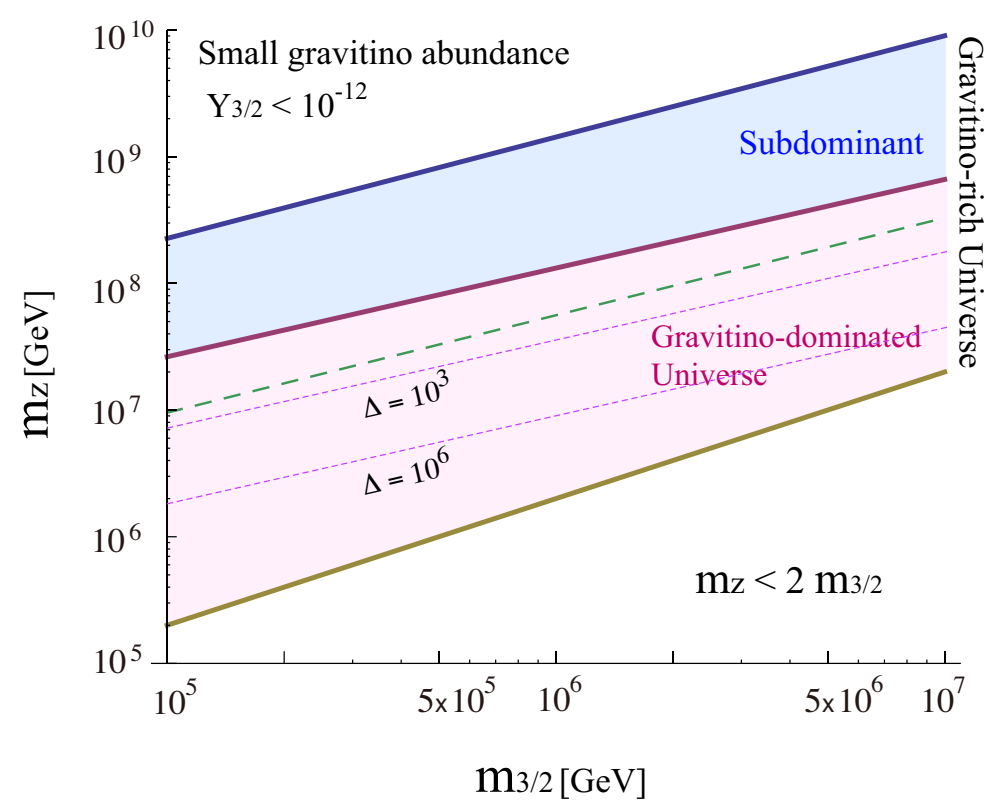

Figure 1: In the shaded (red and blue) regions, the Universe becomes gravitino-rich (see Eq. (11)). The gravitino dominates the Universe in the lower shaded (red) region. The pseudo modulus also dominates the Universe in the region below the dashed line. We have fixed $T_{R}=10^{9} \mathrm{GeV}$, but the lines are only weakly dependent on $T_{R}$. See the text for details.

The quartic coupling in the Kähler potential induces the $z$ decay into the goldstino pair with the decay rate

$$
\Gamma(z \rightarrow \tilde{z} \tilde{z}) \simeq \frac{1}{96 \pi} \frac{m_{z}^{5}}{m_{3 / 2}^{2} M_{P}^{2}} .
$$

As long as $z$ does not have any sizable couplings with the SM sector 3 it predominantly decays into a pair of gravitinos. Thus, the gravitino abundance from the pseudo-modulus decay is given by

$$
Y_{3 / 2}^{(z)} \simeq 6 \times 10^{-7}\left(\frac{T_{R}}{10^{9} \mathrm{GeV}}\right)\left(\frac{m_{3 / 2}}{10^{3} \mathrm{TeV}}\right)^{4}\left(\frac{m_{z}}{10^{8} \mathrm{GeV}}\right)^{-5} .
$$

The Universe becomes gravitino-rich if $Y_{3 / 2}^{(z)}$ is greater than $10^{-12}$, namely,

$$
m_{z} \lesssim 1 \times 10^{9} \mathrm{GeV}\left(\frac{T_{R}}{10^{9} \mathrm{GeV}}\right)^{\frac{1}{5}}\left(\frac{m_{3 / 2}}{10^{3} \mathrm{TeV}}\right)^{\frac{4}{5}}
$$

\footnotetext{
${ }^{3}$ Planck-suppressed couplings do not change the argument.
} 
The gravitino-dominated Universe is realized if

$$
m_{z} \lesssim 1 \times 10^{8} \mathrm{GeV}\left(\frac{g_{*}}{80}\right)^{\frac{1}{20}}\left(\frac{T_{R}}{10^{9} \mathrm{GeV}}\right)^{\frac{1}{5}}\left(\frac{m_{3 / 2}}{10^{3} \mathrm{TeV}}\right)^{\frac{7}{10}} .
$$

In Fig. 1, the Universe becomes gravitino-rich in the shaded (red and blue) regions. In the lower shaded (red) region, the gravitino dominates the Universe, while it does not in the upper shaded (blue) region. The dotted (purple) lines show the contours of the entropy dilution factor, $\Delta=10^{3}$ and $10^{6}$. In the upper left region, the condition (24) is not satisfied and the gravitino abundance is small, while the decay into a pair of gravitinos is kinematically forbidden in the lower right region. In the region below the dashed line, the pseudo modulus dominates the Universe before the decay. We have fixed $T_{R}=10^{9} \mathrm{GeV}$ in the figure, but the conditions on $m_{z}$ are only weakly dependent on $T_{R}$; both the upper and middle solid lines (the dashed line) shift in proportion to $\propto T_{R}^{\frac{1}{5}}\left(T_{R}^{2 / 13}\right)$.

\subsubsection{Modulus field}

Next we consider a modulus field as a source of gravitinos. In superstring theories, moduli fields necessarily appear at low energies through compactifications. Most of these moduli must be stabilized in order to get a sensible low-energy theory, since the moduli determine all the physically relevant quantities such as the size of the extra dimensions, physical coupling constants, and even the SUSY breaking scale. Many moduli fields are stabilized by flux compactifications [34, 35], and the remaining ones can be stabilized a la KKLT [20].

The detailed properties of the modulus depend on the compact geometry, brane configurations, and stabilization mechanism. Here we consider a modulus field $X$ stabilized a la KKLT; it has a mass heavier than the gravitino, and its F-term is suppressed by the mass as $F_{X} \sim m_{3 / 2}\langle X\rangle / m_{X}$, where $m_{X}$ denotes the mass of $X$. During inflation the potential of $X$ is considered to be deformed 4 Then the potential minimum may be deviated from the low-energy minimum, leading to coherent oscillations after inflation. We assume that the energy stored in the coherent oscillations of $X$ dominates the Universe. The entropy density $s_{i}$ should be interpreted as that from the modulus decay in this subsection.

\footnotetext{
${ }^{4}$ There is an upper bound on the inflation scale in order to avoid the destabilization and run-away. Even for the inflation satisfying the bound, the cosmological moduli problem still exists [33.
} 
We also assume that the modulus $X$ has Planck-suppressed couplings to the visible sector so that its decay rate can be expressed as

$$
\Gamma_{X}=\frac{c}{4 \pi} \frac{m_{X}^{3}}{M_{P}^{2}}
$$

where $c$ is a numerical constant of order unity. The decay temperature of the modulus is

$$
\begin{aligned}
T_{X} & =\left(\frac{\pi^{2} g_{*}\left(T_{X}\right)}{90}\right)^{-\frac{1}{4}} \sqrt{\Gamma_{X} M_{P}} \\
& \simeq 3 \mathrm{GeV}\left(\frac{g_{*}\left(T_{X}\right)}{80}\right)^{-\frac{1}{4}}\left(\frac{m_{X}}{10^{7} \mathrm{GeV}}\right)^{\frac{3}{2}} .
\end{aligned}
$$

It was shown in Refs. [8, 9, 10, that such a modulus generically decays into a pair of gravitinos and the branching fraction is sizable: $B_{3 / 2}=\mathcal{O}(0.01-0.1) 5$ Then the gravitino abundance from the modulus decay is estimated as

$$
\begin{aligned}
Y_{3 / 2}^{(X)} & =2 B_{3 / 2} \frac{3}{4} \frac{T_{X}}{m_{X}} \\
& \simeq 6 \times 10^{-7} B_{3 / 2} \sqrt{c}\left(\frac{g_{*}}{80}\right)^{-\frac{1}{4}}\left(\frac{m_{X}}{10^{7} \mathrm{GeV}}\right)^{\frac{1}{2}} .
\end{aligned}
$$

Thus, the Universe becomes gravitino-rich for a wide range of the modulus mass and the branching fraction of the gravitino production. In particular, if the branching fraction into gravitinos is of order 0.1 , the gravitino even dominates the Universe. (Compare (30) with ([6) $)$.)

\subsubsection{Inflaton}

In a series of works $[9,10,11,12,13,14,15,21]$, it was revealed that the inflaton generally decays into gravitinos. The gravitino production rate depends on whether there is a SUSY breaking field that is singlet under any symmetries. Such a singlet is required in the gravity mediation to generate gaugino masses of the correct size, while it is not necessary in the gauge and anomaly mediation. We assume that there is no such a singlet for the moment.

The gravitinos are produced by various processes; (a) the gravitino pair production [9, 10, 11, 12]; (b) decay into the SUSY breaking sector at tree level [13]; (c) anomaly-induced

\footnotetext{
${ }^{5}$ Precisely speaking, this is the case if the SUSY breaking field is heavier than the modulus mass, or if there is a SUSY breaking field that is singlet under any symmetries.
} 
decay into the SUSY breaking sector at one-loop level [14]. The gravitino production rate can be expressed as

$$
\Gamma_{3 / 2}=\frac{x}{32 \pi}\left(\frac{\langle\phi\rangle}{M_{P}}\right)^{2} \frac{m_{\phi}^{3}}{M_{P}^{2}}
$$

where $m_{\phi}$ is the inflaton mass, and $\langle\phi\rangle$ a VEV of the inflaton. Here it should be noted that $\langle\phi\rangle$ is evaluated at the potential minimum after inflation. The precise value of the numerical coefficient $x$ depends on the production processes, possible non-renormalizable couplings in the Kähler potential, and the details of the SUSY breaking models [15]. To be concrete, let us assume the minimal Kähler potential and the dynamical SUSY breaking (DSB) with a dynamical scale $\Lambda$. As we have seen before, the SUSY breaking field $z$ can acquire a mass $m_{z}$ heavier than $m_{3 / 2}$, and we assume $m_{z} \sim \Lambda \sim \sqrt{m_{3 / 2} M_{P}}$ in the following 6

For a low-scale inflation model with $m_{\phi}<\Lambda$, the process (a) becomes effective, and $x=1$. On the other hand, for the inflaton mass larger than $\Lambda$, the processes (b) and (c) become effective instead. The inflaton decays into the hidden quarks in the SUSY breaking sector via Yukawa couplings of the hidden particles (process (b)), or into the hidden gauge sector via anomalies (process (c)). Since the hidden quarks and gauge bosons (and gauginos) are energetic when they are produced, they will form jets and produce hidden hadrons through the strong gauge interactions. The gravitinos are likely produced by the decays of the hidden hadrons. We denote the averaged number of the gravitinos produced per each jet as $N_{3 / 2}$. Then $x$ is given by [15]

$$
x \simeq \frac{N_{3 / 2}}{8 \pi^{2}}\left(\frac{1}{2} N_{y}\left|Y_{h}^{2}\right|+N_{g} \alpha_{h}^{2}\left(T_{g}^{(h)}-T_{r}^{(h)}\right)^{2}\right),
$$

where $Y_{h}$ and $\alpha_{h}$ are the Yukawa coupling and a fine structure constant of the hidden gauge group, respectively, $N_{y}$ denotes a number of the final states for the process (b), $N_{g}$ is a number of the generators of the gauge group, and $T_{g}^{(h)}$ and $T_{r}^{(h)}$ are the Dynkin indices of the adjoint representation and the matter fields in the representation $r$. Although $x$ depends on the structure of the SUSY breaking sector, its typical magnitude is $O\left(10^{-2}-\right.$

\footnotetext{
${ }^{6}$ The gravitino production can be suppressed if the SUSY breaking field is lighter than the inflaton and the inflaton mass is below the dynamical scale [21, 36].
} 


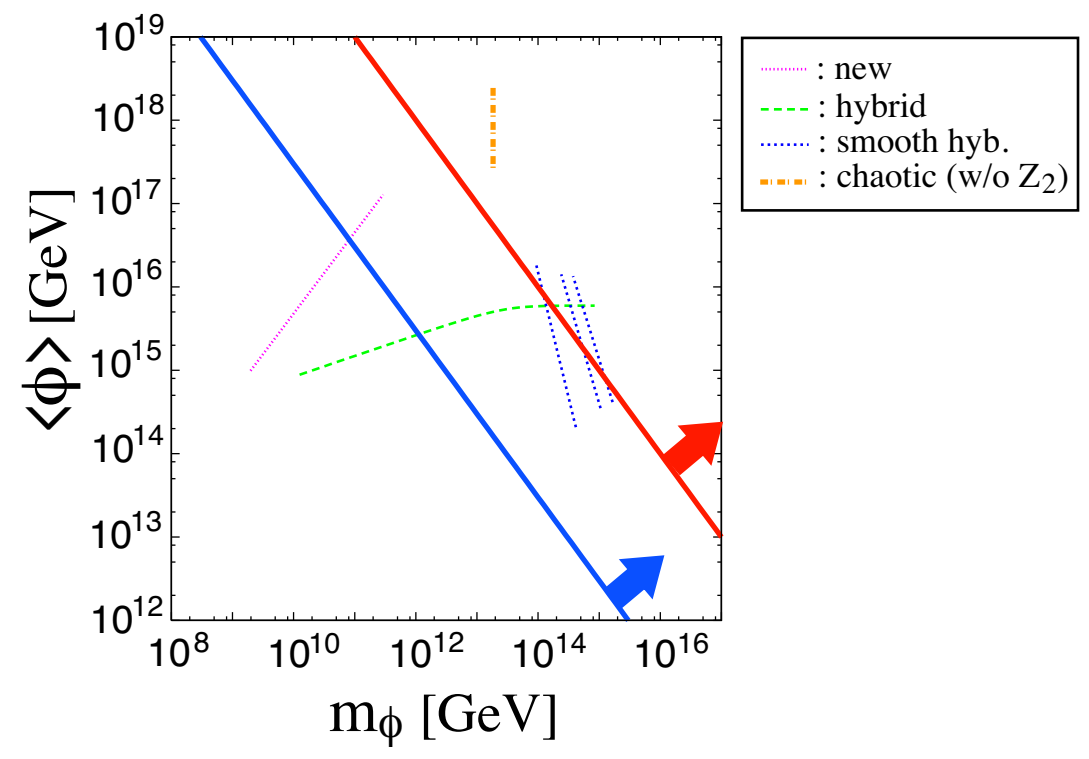

Figure 2: The gravitino-rich Universe is realized in the region above the blue solid line, while the gravitino dominates the Universe in the region above the red solid line. We have set $x=1, T_{R}=10^{9} \mathrm{GeV}$ and $m_{3 / 2}=10^{3} \mathrm{TeV}$.

10) for $m_{\phi}>\Lambda 7$ To be concrete we adopt $x=1$ as a reference value in the following, but it should be kept in mind that there are uncertainties in the gravitino production rate in this case.

The abundance of gravitinos from the inflaton decay is therefore

$$
\begin{aligned}
Y_{3 / 2} & =\frac{\Gamma_{3 / 2}}{\Gamma_{\phi}} \frac{3}{4} \frac{T_{R}}{m_{\phi}} \\
& \simeq 1 \times 10^{-7} x\left(\frac{g_{*}\left(T_{R}\right)}{200}\right)^{-\frac{1}{2}}\left(\frac{\langle\phi\rangle}{10^{15} \mathrm{GeV}}\right)^{2}\left(\frac{m_{\phi}}{10^{15} \mathrm{GeV}}\right)^{2}\left(\frac{T_{R}}{10^{9} \mathrm{GeV}}\right)^{-1} .
\end{aligned}
$$

Thus, the gravitino abundance increases as the inflaton VEV and mass. Also, it should be noted that the gravitino abundance is inversely proportional to the reheating temperature.

The Universe will be gravitino-rich, if

$$
\langle\phi\rangle>3 \times 10^{12} \mathrm{GeV} x^{-\frac{1}{2}}\left(\frac{g_{*}\left(T_{R}\right)}{200}\right)^{\frac{1}{4}}\left(\frac{m_{\phi}}{10^{15} \mathrm{GeV}}\right)^{-1}\left(\frac{T_{R}}{10^{9} \mathrm{GeV}}\right)^{\frac{1}{2}},
$$

\footnotetext{
${ }^{7}$ Roughly, we expect $N_{3 / 2}=O\left(1-10^{2}\right), N_{g}=O(1), \alpha_{h}=0.1-1$, and $T_{g}^{(h)}-T_{r}^{(h)}=O(1)$, while $Y_{h}$ strongly depends on the SUSY breaking models.
} 
and the gravitino dominates the Universe if

$$
\begin{aligned}
\langle\phi\rangle> & 1 \times 10^{15} \mathrm{GeV} x^{-\frac{1}{2}}\left(\frac{g_{*}\left(T_{3 / 2}\right)}{80}\right)^{-\frac{1}{8}}\left(\frac{g_{*}\left(T_{R}\right)}{200}\right)^{\frac{1}{4}}\left(\frac{m_{3 / 2}}{10^{3} \mathrm{TeV}}\right)^{\frac{1}{4}} \\
& \times\left(\frac{m_{\phi}}{10^{15} \mathrm{GeV}}\right)^{-1}\left(\frac{T_{R}}{10^{9} \mathrm{GeV}}\right)^{\frac{1}{2}} .
\end{aligned}
$$

The regions satisfying (35) or (36) are shown in Fig. 2, together with representative inflation models, new [37, 38, hybrid [39], smooth hybrid [40] and chaotic [41] inflation. (The effect of the constant term in the superpotential on the inflaton dynamics [42, 43] is not taken into account in this figure.) We have set $x=1, T_{R}=10^{9} \mathrm{GeV}$ and $m_{3 / 2}=$ $10^{3} \mathrm{TeV}$. One can see that the Universe can be gravitino-rich for a large portion of the parameter space, and this region will be larger for lower $T_{R}$. For instance, in the smooth hybrid inflation, the inflaton mass and VEV are of order $10^{15} \mathrm{GeV}$. In the chaotic inflation without a $Z_{2}$ symmetry, the inflaton mass is about $2 \times 10^{13} \mathrm{GeV}$, and the VEV is around the Planck scale. For such high-scale inflation models, the resultant gravitino abundance is so large that the gravitino dominated Universe can be realized.

So far, we have assumed the absence of the SUSY breaking singlet field $z$. When it is present, it generally enhances the gravitino production rate through the operators like $K \supset|\phi|^{2} z$, or $|\phi|^{2} z z$. The former induces a kinetic mixing between the inflaton and $z$, while the latter induces the pair goldstino production. Furthermore, such a singlet $z$ may be produced as coherent oscillations, and it decays into gravitinos. Thus, the gravitino-rich Universe will be more plausible in the presence of such a singlet.

\section{Cosmology}

In this section we discuss cosmology of the gravitino-rich Universe. In particular we study the neutralino DM produced by the gravitino decay, and estimate the baryon asymmetry in thermal leptogenesis in the presence of entropy dilution.

\subsection{Neutralino dark matter abundance}

We assume that the lightest neutralino $\chi$ is the LSP. It decouples from thermal bath at the freeze-out temperature, $T_{f} \sim m_{\chi} / 28$ for the neutralino mass $m_{\chi}$ around or above 
a few hundred GeV. Since $T_{3 / 2}$ is lower than $T_{f}$ for the gravitino at the PeV scale, the neutralino abundance produced by gravitino decay is determined according to the Boltzmann equation,

$$
\frac{d n_{\chi}}{d t}+3 H n_{\chi}=-\left\langle\sigma_{\chi} v_{\mathrm{rel}}\right\rangle n_{\chi}^{2}
$$

where $n_{\chi}$ is the number density of $\chi$. The effective annihilation cross section of the lightest neutralino is parameterized by

$$
\left\langle\sigma_{\chi} v_{\mathrm{rel}}\right\rangle=\frac{c_{\chi}}{m_{\chi}^{2}}
$$

One then finds the relic abundance of $\chi$ to be [44, 45]

$$
\left(\frac{n_{\chi}}{s}\right)^{-1}=\left.\left(\frac{n_{\chi}}{s}\right)^{-1}\right|_{T=T_{3 / 2}}+\left.\left(\frac{H}{s\left\langle\sigma_{\chi} v_{\mathrm{rel}}\right\rangle}\right)^{-1}\right|_{T=T_{3 / 2}} .
$$

When the gravitino abundance is large enough, the annihilation among the neutralinos will take place until the expansion rate $H$ becomes equal to the annihilation rate. This results in

$$
\frac{n_{\chi}}{s} \simeq 2.1 \times 10^{-12}\left(\frac{g_{*}\left(T_{3 / 2}\right)}{80}\right)^{-1 / 4}\left(\frac{c_{\chi}}{10^{-2}}\right)^{-1}\left(\frac{m_{\chi}}{300 \mathrm{GeV}}\right)^{2}\left(\frac{m_{3 / 2}}{10^{3} \mathrm{TeV}}\right)^{-3 / 2}
$$

for $Y_{3 / 2} \gtrsim 10^{-12}$.

In the gravitino-rich Universe, the lightest neutralino needs to have a large annihilation cross section in order not to overclose the Universe. This implies that, even if there are neutralinos thermally produced before the gravitino decay, their thermal relic density will be small. Let us consider the case where $\chi$ is dominated by the neutral Wino 8 The Wino-like neutralino has $c_{\chi}$ around $10^{-2}$, where we include the coannihilation effect but it is not so large because $T_{3 / 2}$ is similar in size to the mass difference of the charged and neutral Winos. Fig. 3 shows the parameter region where the Wino-like neutralinos from gravitino decay account for the observed DM density. For $Y_{3 / 2} \gtrsim 10^{-12}$, the neutral Wino with mass of several hundred $\mathrm{GeV}$ can constitute the DM of the Universe. We also plot the contours of the entropy dilution factor $\Delta$ for the gravitino-domination case,

8 See Ref. [46] for the higgsino DM in the heavy gravitino scenario, and also Ref. [47, 48, for the neutralino DM from gravitino decay in the case with $Y_{3 / 2}$ less than $10^{-12}$. 


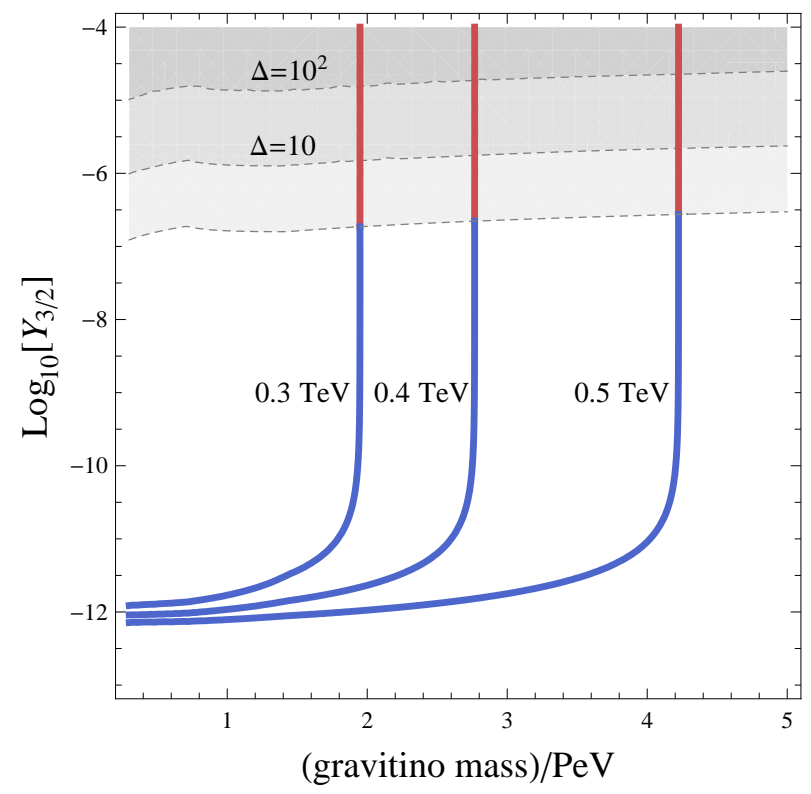

Figure 3: Wino DM density from gravitino decay consistent with the observation, for $m_{\chi}=0.3,0.4,0.5 \mathrm{TeV}$, respectively. The left side of the solid line for given $m_{\chi}$ is excluded by the overclosure constraint. The gravitino dominates the Universe in the shaded region.

$Y_{3 / 2} \gtrsim 10^{-7}$. One can see from the figure that the Wino DM density is independent of the gravitino abundance in the gravitino-rich Universe, and that the correct DM abundance can be explained for the Wino-like neutralino with mass of several hundred $\mathrm{GeV}$ and the gravitino at the $\mathrm{PeV}$ scale. In the next section we will discuss how to realize such hierarchy between the gaugino mass and the gravitino mass.

Meanwhile, since the charged Wino is nearly degenerated in mass with the neutral Wino, Sommerfeld effect should be included when the Wino has mass around $4 \pi m_{W} / g^{2} \sim$ $2 \mathrm{TeV}$ [49]. However the resulting annihilation cross section would not be sufficiently large to avoid the overclosure constraint for the gravitino mass at the PeV scale.

\subsection{Baryon asymmetry in leptogenesis}

One plausible way to generate the baryon asymmetry is through leptogenesis [50], and the simplest realization is to use lepton number violation of the right-handed neutrinos in the seesaw mechanism [51]. Here we consider thermal leptogenesis in the gravitino-rich Universe. 
The observed baryon asymmetry of the Universe can be explained through thermal leptogenesis if the reheating temperature after inflation is high enough, $T_{R} \gtrsim 10^{9} \mathrm{GeV}$ [52]. On the other hand, the gravitino abundance increases in proportional to $T_{R}$ when produced by thermal scattering or pseudo-modulus decay. Hence one may worry about the overproduction of DM by the gravitino decay for the reheating temperature required by the successful thermal leptogenesis. In order to avoid the tension, $T_{R}$ must be chosen to be close to about $10^{9-10} \mathrm{GeV}$ for $m_{3 / 2}=10 \mathrm{TeV}-100 \mathrm{TeV} 9$

Such a tension can be relaxed if the neutralino LSP has mass of several hundred $\mathrm{GeV}$, while the gravitino mass is at the PeV scale, as the neutralino DM abundance becomes independent of the gravitino abundance. In addition, if the gravitino dominates the Universe, there is an entropy dilution. When the gravitinos are produced by thermal scatterings or the pseudo-modulus decay, the entropy dilution factor is approximately proportional to the reheating temperature, implying that the present baryon asymmetry reads

$$
\frac{n_{B}}{s} \simeq 3 \times 10^{-10} \Delta^{-1}\left(\frac{\kappa}{0.1}\right)\left(\frac{M_{1}}{10^{10} \mathrm{GeV}}\right)\left(\frac{m_{\nu 3}}{0.05 \mathrm{eV}}\right) \delta_{\mathrm{eff}} \propto \frac{M_{1}}{T_{R}}
$$

where $\kappa$ denotes the efficiency factor, $m_{\nu 3}$ the heaviest neutrino mass, and $\delta_{\text {eff }}$ the effective CP phase. Here we consider the lepton asymmetry generated by the decay of the lightest right-handed neutrino with mass $M_{1}\left(\lesssim T_{R}\right)$, which is converted to the baryon asymmetry via the sphaleron effect. Noting that the maximum value of the baryon asymmetry is realized for $M_{1} \sim T_{R}$, and that the dilution factor scales as $\Delta \propto T_{R}$, one can see that the maximum value of baryon asymmetry from leptogenesis will be independent of $T_{R}$.

Thus, if the gravitino-dominant Universe and the mass hierarchy between the LSP and the gravitino are realized, both the maximum baryon asymmetry in thermal leptogenesis and the neutralino DM abundance are independent of $T_{R}$. This feature has an interesting implication that thermal leptogenesis can be implemented at high reheating temperatures while avoiding overproduction of DM.

Let us see the case where the gravitino produced by pseudo-modulus decay dominates the Universe. In Fig. 4, we show the contours of Wino DM density consistent with the

\footnotetext{
${ }^{9}$ Interestingly, the neutrino mass anarchy and the observed neutrino mass squared differences suggest $T_{R}=10^{9} \mathrm{GeV}-10^{10} \mathrm{GeV}$, when combined with thermal leptogenesis. 53 ]
} 


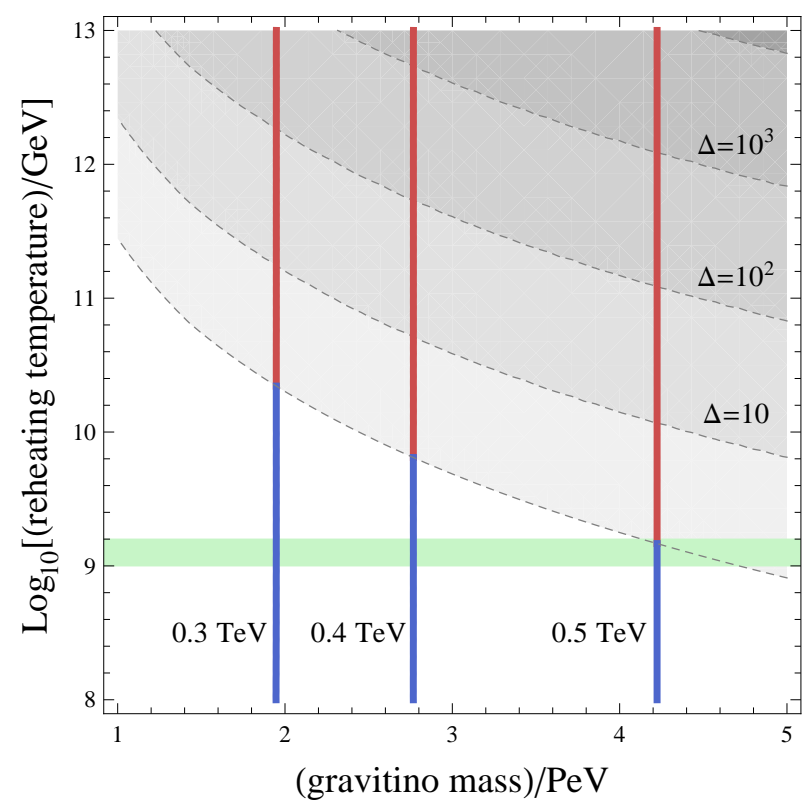

Figure 4: Gravitino-rich Universe in the pseudo-modulus scenario with $m_{z}=4 \times 10^{8} \mathrm{GeV}$. The solid lines correspond to the contours of Wino DM density from gravitino decay consistent with the observation, for $m_{\chi}=0.3,0.4,0.5 \mathrm{TeV}$, respectively. The gravitino dominates the Universe in the shaded region. For successful thermal leptogenesis, the reheating temperature after inflation should be higher than about $10^{9} \mathrm{GeV}$. The maximum value of the baryon asymmetry is constant along the (red) solid line in the shaded region.

observation in the $\left(m_{3 / 2}, T_{R}\right)$ plane, for the pseudo-modulus with mass $m_{z}=4 \times 10^{8} \mathrm{GeV}$. Along the solid (red) lines in the shaded region, the gravitino dominates the Universe, and the maximum value of the baryon asymmetry in thermal leptogenesis is constant because $\Delta \propto T_{R}$. For heavier (lighter) $m_{z}$, the pseudo-modulus abundance decreases, and the contour of the entropy dilution factor will shift upwards (downwards).

\section{Mass hierarchy between gaugino and gravitino}

We have seen in the previous section that the Wino-like neutralino LSP of mass several hundred $\mathrm{GeV}$ produced by the decay of the gravitino at the PeV scale can account for the observed DM density. In this section we discuss how to realize such mass hierarchy between the Wino-like neutralino and the gravitino.

So far we have not specified how the SUSY breaking is mediated to the visible sector. 
In the gravity mediation, both gaugino and scalar masses arise from coupling with a SUSY breaking singlet, and all the superparticles have masses comparable to the gravitino mass. In this case, the Wino mass can be much lighter than the other SUSY particles only at the price of fine-tuning the coupling. Since the naturalness is not a serious issue in the high-scale SUSY, we cannot exclude this possibility, and indeed such fine-tuning may be required by the anthropic condition on the DM abundance.

In the absence of such SUSY breaking singlet, on the other hand, the gaugino mass can be hierarchically lighter than the gravitino mass. In the heavy gravitino scenario, the anomaly mediation gives dominant contributions to the gaugino mass [54, 55]:

$$
M_{\lambda} \sim \frac{m_{3 / 2}}{8 \pi^{2}}
$$

unless one considers a specific SUSY breaking scenario such as no-scale type models. Scalar masses are more model-dependent; the scalar fields obtain masses $\sim m_{3 / 2}$ through contact interactions with a SUSY breaking field in the Kähler potential, which are allowed even if the SUSY breaking field is charged under some symmetry. However, if the visible sector is dynamically sequestered from the SUSY breaking sector, it is possible that the scalar masses are suppressed. Compared to the other soft terms, therefore, the gaugino masses are less model-dependent, and have a simple form [56].

To arrange a light Wino of mass several hundred $\mathrm{GeV}$ for the gravitino mass at $\mathrm{PeV}$ scale, the anomaly-mediation relation (42) is not sufficient, and one needs further suppression 10 One way is to introduce SM vector-like matter fields $\Psi+\bar{\Psi}$ which obtain masses from the VEV of a SM singlet so that their loops generate soft masses via gauge mediation. Let us consider $N_{\Psi}$ pairs of $\Psi+\bar{\Psi}$ forming $\mathbf{5}+\overline{\mathbf{5}}$ representation of $\mathrm{SU}(5)$ with

$$
W=y_{\Psi} S \Psi \bar{\Psi}+f(S)
$$

for which the gauge coupling unification of the MSSM is not spoiled. Including the contribution from the messenger loops, low energy gaugino masses read

$$
\frac{M_{a}}{g_{a}^{2}}=\frac{b_{a}}{16 \pi^{2}} m_{3 / 2}-\frac{N_{\Psi}}{16 \pi^{2}} \frac{F^{S}}{S},
$$

\footnotetext{
${ }^{10}$ In no-scale models, the chiral compensator auxiliary field is much suppressed compared to $m_{3 / 2}$, and consequently anomaly-mediated soft masses are much smaller than $m_{3 / 2} / 8 \pi^{2}$. This provides another way to have relatively light gauginos in the heavy gravitino scenario.
} 
where $\left(b_{1}, b_{2}, b_{3}\right)=(33 / 5,1,-3)$ are the MSSM beta function coefficients.

The anomaly-mediated Wino mass can be cancelled when $S$ is stabilized in such a way that $\left(F^{S} / S\right) / m_{3 / 2}$ is positive and order unity. A simple way to achieve this is to consider a composite $S$ having an Affleck-Dine-Seiberg superpotential [57],

$$
f(S)=\frac{\Lambda_{c}^{3+n}}{S^{n}}
$$

where $\Lambda_{c}$ is the dynamical scale much lower than the GUT scale, and $n$ is a positive rational number. Then the $F$-term scalar potential from the above superpotential competes with the associated $A$-term to fix $S$ at $\left(\Lambda_{c} / m_{3 / 2}\right)^{1 /(n+2)} \Lambda_{c}$ with

$$
\frac{F^{S}}{S}=\frac{2}{n+1}\left(1+\mathcal{O}\left(\frac{m_{S}^{2}}{m_{3 / 2}^{2}}\right)\right) m_{3 / 2},
$$

where $m_{S}^{2}$ is the soft mass squared of $S$. Assuming that $S$ acquires soft mass around $m_{3 / 2} / 8 \pi^{2}$, one finds that the Bino and Gluino have masses around $m_{3 / 2} / 8 \pi^{2}$ whereas the Wino obtains a relatively light mass for $n=2 N_{\Psi}-1$ :

$$
M_{\tilde{W}} \sim \frac{m_{3 / 2}}{\left(8 \pi^{2}\right)^{2}}
$$

which is a few hundred $\mathrm{GeV}$ for the gravitino at the $\mathrm{PeV}$ scale. Since $S$ is a composite, the messengers have a small Yukawa coupling $y_{\Psi} \sim \Lambda_{c} / M_{P}$, and get a SUSY preserving mass $M_{\Psi} \sim\left(\Lambda_{c} / m_{3 / 2}\right)^{1 /(n+2)} \Lambda_{c}^{2} / M_{P}$. Thus a dynamical scale higher than about $10^{12} \mathrm{GeV}$ leads to $M_{\Psi} \gg m_{3 / 2}$ as required for the relation (44) to be valid.

Alternatively, in order to fix $S$ at a vacuum giving a positive $\left(F^{S} / S\right) / m_{3 / 2}$, one may consider the model with

$$
\begin{aligned}
K & =|Z|^{2}+\sum_{\Phi}\left(|\Phi|^{2}+\frac{\kappa_{\Phi}}{3} \frac{|Z|^{2}}{M_{P}^{2}}|\Phi|^{2}\right), \\
f(S) & =y \Sigma\left(M^{2}-S S^{\prime}\right),
\end{aligned}
$$

for $\Phi=\left\{\Sigma, S, S^{\prime}, \Psi, \bar{\Psi}\right\}$ and $y M \gg m_{3 / 2}$, so that $S$ is stabilized near the $F$-flat direction, $S S^{\prime}=M^{2}$. Here $Z$ is the SUSY breaking field with $F$-term $\sim m_{3 / 2} M_{P}$, and $\kappa_{\Phi}=1$ if $\Phi$ resides in a sector sequestered from the SUSY breaking sector. For $y_{\Psi}$ of order unity, the ratio between the soft scalar masses of $S$ and $S^{\prime}$ is naively estimated by

$$
\frac{m_{S^{\prime}}^{2}}{m_{S}^{2}} \sim \frac{\left(8 \pi^{2}\right)^{2}\left(1-\kappa_{S^{\prime}}\right)+y^{2}}{\left(8 \pi^{2}\right)^{2}\left(1-\kappa_{S}\right)+y_{\Psi}^{2}} .
$$


Let us assume $m_{S}^{2} \gg m_{S^{\prime}}^{2}$, which would be the case when $\kappa_{S}$ is less than one while $\kappa_{\Phi}=1$ for the others, or when $\kappa_{\Phi}=1$ for all the involved fields and $y \ll y_{\Psi}$. Then the scalar potential develops a minimum at $\left|S^{\prime}\right|^{2} \simeq\left(m_{S}^{2} / m_{S^{\prime}}^{2}\right) M^{2} \gg|S|^{2}$ with

$$
\frac{F^{S}}{S}=\left(1+\mathcal{O}\left(\frac{m_{S^{\prime}}^{2}}{m_{S}^{2}}\right)\right) m_{3 / 2},
$$

and therefore the anomaly-mediated Wino mass is cancelled for $N_{\Psi}=1$, making it possible to have $M_{\tilde{W}} \sim m_{3 / 2} /\left(8 \pi^{2}\right)^{2}$ for $m_{S}^{2}$ larger than about $8 \pi^{2} m_{S^{\prime}}^{2}$. Meanwhile, the messenger mass reads $M_{\Psi} \sim\left(m_{S^{\prime}}^{2} / m_{S}^{2}\right)^{1 / 2} M$.

It is interesting to note that the above model possesses a global $\mathrm{U}(1)$, under which $S$ and $S^{\prime}$ have charges of opposite sign while $\Sigma$ is neutral. This can be identified as the Peccei-Quinn (PQ) symmetry solving the strong CP problem [58, 59], with the axion scale fixed at $F_{a} \sim\left|S^{\prime}\right|$. The axion also constitutes the DM with $\Omega_{a} \sim 0.4 \theta_{i}^{2}\left(F_{a} / 10^{12} \mathrm{GeV}\right)^{1.18}$ where $\theta_{i}$ is the initial misalignment. The axion relic energy density will be diluted if the gravitino decays after the QCD phase transition, for which $F_{a}$ larger than $10^{12} \mathrm{GeV}$ is allowed even for large initial misalignment.

Let us move to the possibility of arranging a light Wino through the higgsino loops. Including higgsino threshold effects, the gaugino masses are written [55, 60]

$$
\frac{M_{a}}{g_{a}^{2}}=\frac{b_{a}}{16 \pi^{2}} m_{3 / 2}+\alpha \frac{k_{a}}{16 \pi^{2}} m_{3 / 2},
$$

for $\left(k_{1}, k_{2}, k_{3}\right)=(3 / 5,1,0)$, and $\alpha$ defined by

$$
\alpha=\frac{\mu \sin 2 \beta}{m_{3 / 2}} \frac{m_{A}^{2}}{|\mu|^{2}-m_{A}^{2}} \ln \left(\frac{|\mu|^{2}}{m_{A}^{2}}\right),
$$

where $\mu$ is the higgsino mixing parameter, $m_{A}$ is the mass of the heavy Higgs bosons, and $\tan \beta$ is the ratio of the Higgs VEVs. For a large $\mu$ around $m_{3 / 2}$ and low $\tan \beta$, the higgsino correction can cancel the anomaly-mediated Wino mass, leading to a light Wino at the price of fine-tuning.

Finally we briefly discuss phenomenological aspects of the model, where the lightest neutralino $\chi$ and the lightest chargino $\chi^{ \pm}$are both Wino-like. For large $\mu$, the dominant mass splitting between $\chi$ and $\chi^{ \pm}$comes from gauge boson loops, and makes the chargino heavier than the neutralino by about $160 \mathrm{MeV}$ for $M_{\tilde{W}}$ around several hundred $\mathrm{GeV}$ 
[61. Hence $\chi^{ \pm}$dominantly decays into $\chi$ plus soft $\pi^{ \pm}$with a decay length of a few $\mathrm{cm}$, or longer if boosted, while producing a visible track in the detector. Meanwhile, there are cosmological and astrophysical constraints arising since the neutral Wino has a large annihilation cross section into a W-boson pair, which require the Wino mass to be larger than about $300 \mathrm{GeV}[62$.

\section{Discussion and Conclusions}

Being the superpartner of the graviton, the gravitino is coupled to all the sectors with Planck-suppressed interactions. Thus, once the gravitino dominates the Universe, it decays into all the lighter degrees of freedom, including those in the visible sector. Then the question is how to generate the baryon asymmetry and DM. In Sec 4 we have considered thermal leptogenesis and the Wino-like neutralino LSP non-thermally produced by the gravitino decay. Here we briefly discuss other possibilities.

One is the so-called gravitino-induced baryogenesis [63]. In the presence of an R-parity and baryon-number violating operator, $U_{i} D_{j} D_{k}$, and the associated $A$-term with a CP phase, the gravitino decay can generate a right amount of the baryon asymmetry. Since the coefficient of the R-parity violating operator must be sizable for the mechanism to work, any pre-existing baryon asymmetry would be washed out in this scenario. Also, we need a source of CP violation; if there is a SUSY breaking singlet field, it can satisfy the requirement. The presence of such SUSY breaking singlet generally enhances the gravitino production from the inflaton decay, and furthermore, its coherent oscillations can decay into gravitinos. Thus, the presence of the SUSY breaking singlet makes both the gravitino dominance and the gravitino-induced baryogenesis plausible.

Another one is the Affleck-Dine mechanism [64]. The AD mechanism is so efficient that a sufficient amount of baryon asymmetry can be generated even in the presence of entropy production by the gravitino decay. The AD mechanism in high-scale SUSY was explored in detail in Ref. [33].

One of the plausible candidate for DM is the QCD axion. If the gravitino decays after the QCD phase transition, the QCD axion abundance is diluted, allowing a larger value of the axion decay constant 65. 
So far we have assumed that the gravitino is heavier than all the MSSM particles. However, it is also possible that the gravitino decay into some of the MSSM particles is kinematically forbidden. In this case the gravitino lifetime becomes longer, and the gravitino mass is required to be heavier in order to avoid the tight BBN bound.

If there are additional light degrees of freedom such as the axion and axino, or any light degrees of freedom in the hidden sector, the gravitino will decay into those degrees of freedom. The branching fraction into these hidden particles can be sizable especially if some of the MSSM particles are heavier than the gravitino. Interestingly, there is an argument that the string theory contains a plenitude of string axions [66]. If the gravitino decays into those axions and axinos with a sizable branching fraction, the produced axions (and axinos) can account for the dark radiation hinted by the recent observations [24]. Also, if some of the hidden sector particles thus produced are stable in a cosmological time scale, they will contribute to the DM; depending on its mass it can be hot or warm DM.

In this paper, we have studied the gravitino cosmology in high-scale SUSY as suggested by the recently discovered Higgs boson with mass $125-126 \mathrm{GeV}$. We have discussed various gravitino production processes such as thermal production as well as non-thermal production by the (pseudo) modulus and inflaton decay, in order to see if the gravitinos are abundantly produced in the early Universe. We have shown that the Universe can be gravitino-rich for a wide range of parameters, and it is even possible for the gravitino to dominate the Universe. In the gravitino-rich Universe, the neutralino LSP must have a large annihilation cross section, since otherwise the LSPs produced by the gravitino decay would overclose the Universe. We focused on the Wino-like LSP and estimated its abundance as a function of the gravitino mass and abundance. We also discussed how to realize the required mass hierarchy between the lightest neutralino with $m_{\chi}=\mathcal{O}(100) \mathrm{GeV}$ and the gravitino at the PeV scale.

Among various possibilities, we have found that the pseudo-modulus in the SUSY breaking sector is a plausible and interesting source of gravitinos. The dilution factor by the gravitino decay is proportional to the reheating temperature in this case, which makes the maximum value of the baryon asymmetry in thermal leptogenesis independent of the reheating temperature. 
If high-scale SUSY is realized in nature, perhaps the naturalness argument is not the right guiding principle to understand the observable parameters in our Universe. The SUSY breaking scale suggested by the SM-like Higgs boson mass ranges from $10 \mathrm{TeV}$ up to $\mathrm{PeV}$ or even higher, but we do not know the reason why it takes such a value. It may be due to a competition between the bias in the landscape and the anthropic condition such as inflation [38] or entropy dilution by the modulus decay [67].

The gravitino, being the superpartner of the graviton, is long-lived and has universal Planck-suppressed couplings. There are various processes in which gravitinos are produced. Therefore the gravitino likely plays an important role in cosmology, especially if high-scale SUSY is realized. Our Universe might have been gravitino-rich.

\section{Acknowledgment}

This work was supported by the Grant-in-Aid for Scientific Research on Innovative Areas (No.24111702, No. 21111006, and No.23104008) [FT], Scientific Research (A) (No. 22244030 and No.21244033 [FT]), and JSPS Grant-in-Aid for Young Scientists (B) (No. 24740135) [FT]. This work was also supported by World Premier International Center Initiative (WPI Program), MEXT, Japan, and by Grants-in-Aid for Scientific Research from the Ministry of Education, Science, Sports, and Culture (MEXT), Japan (No. 23104008 and No. $23540283[\mathrm{KSJ}])$.

\section{References}

[1] G. Aad et al. [ATLAS Collaboration], Phys. Lett. B 716, 1 (2012) arXiv:1207.7214 [hep-ex]].

[2] S. Chatrchyan et al. [CMS Collaboration], Phys. Lett. B 716, 30 (2012) arXiv:1207.7235 [hep-ex]].

[3] Y. Okada, M. Yamaguchi and T. Yanagida, Phys. Lett. B 262, 54 (1991); see also Y. Okada, M. Yamaguchi and T. Yanagida, Prog. Theor. Phys. 85, 1 (1991); J. R. Ellis, G. Ridolfi and F. Zwirner, Phys. Lett. B 257, 83 (1991); H. E. Haber and R. Hempfling, Phys. Rev. Lett. 66, 1815 (1991). 
[4] G. F. Giudice and A. Strumia, Nucl. Phys. B 858, 63 (2012) arXiv:1108.6077 [hep$\mathrm{ph}]]$;

G. Degrassi, S. Di Vita, J. Elias-Miro, J. R. Espinosa, G. F. Giudice, G. Isidori and A. Strumia, JHEP 1208, 098 (2012) [arXiv:1205.6497 [hep-ph]];

see also F. Bezrukov, M. Y. .Kalmykov, B. A. Kniehl and M. Shaposhnikov, arXiv:1205.2893 [hep-ph].

[5] M. Bolz, A. Brandenburg and W. Buchmuller, Nucl. Phys. B 606, 518 (2001) [Erratum-ibid. B 790, 336 (2008)] [hep-ph/0012052].

[6] J. Pradler and F. D. Steffen, Phys. Rev. D 75, 023509 (2007) hep-ph/0608344; Phys. Lett. B 648, 224 (2007) hep-ph/0612291.

[7] V. S. Rychkov and A. Strumia, Phys. Rev. D 75, 075011 (2007) hep-ph/0701104.

[8] M. Endo, K. Hamaguchi and F. Takahashi, Phys. Rev. Lett. 96, 211301 (2006);

S. Nakamura and M. Yamaguchi, Phys. Lett. B 638, 389 (2006).

[9] M. Dine, R. Kitano, A. Morisse and Y. Shirman, Phys. Rev. D 73, 123518 (2006).

[10] M. Endo, K. Hamaguchi and F. Takahashi, Phys. Rev. D 74, 023531 (2006).

[11] M. Kawasaki, F. Takahashi and T. T. Yanagida, Phys. Lett. B 638, 8 (2006) hep-ph/0603265; Phys. Rev. D 74, 043519 (2006) hep-ph/0605297.

[12] T. Asaka, S. Nakamura and M. Yamaguchi, Phys. Rev. D 74, 023520 (2006) hep-ph/0604132.

[13] M. Endo, M. Kawasaki, F. Takahashi and T. T. Yanagida, Phys. Lett. B 642, 518 (2006) hep-ph/0607170].

[14] M. Endo, F. Takahashi and T. T. Yanagida, Phys. Lett. B 658, 236 (2008) hep-ph/0701042.

[15] M. Endo, F. Takahashi and T. T. Yanagida, Phys. Rev. D 76, 083509 (2007) arXiv:0706.0986 [hep-ph]].

[16] S. Weinberg, Phys. Rev. Lett. 48, 1303 (1982).

[17] J. R. Ellis, A. D. Linde and D. V. Nanopoulos, Phys. Lett. B 118, 59 (1982).

[18] L. M. Krauss, Nucl. Phys. B 227, 556 (1983). 
[19] M. Kawasaki, K. Kohri and N. Sugiyama, Phys. Rev. Lett. 82, 4168 (1999); M. Kawasaki, K. Kohri and N. Sugiyama, Phys. Rev. D 62, 023506 (2000); S. Hannestad, Phys. Rev. D 70, 043506 (2004); K. Ichikawa, M. Kawasaki and F. Takahashi, Phys. Rev. D 72, 043522 (2005).

[20] S. Kachru, R. Kallosh, A. D. Linde and S. P. Trivedi, Phys. Rev. D 68, 046005 (2003) hep-th/0301240].

[21] M. Endo, F. Takahashi and T. T. Yanagida, Phys. Rev. D 76, 083508 (2007) hep-ph/0702247 [HEP-PH]].

[22] E. Komatsu et al. [WMAP Collaboration], Astrophys. J. Suppl. 192 (2011) 18 arXiv:1001.4538 [astro-ph.CO]].

[23] Astrophys. J. 739, 52 (2011). arXiv:1009.0866 [astro-ph.CO]].

[24] K. Ichikawa, M. Kawasaki, K. Nakayama, M. Senami and F. Takahashi, JCAP 0705, 008 (2007) [hep-ph/0703034 [hep-ph]].

[25] J. Jaeckel, J. Redondo and A. Ringwald, Phys. Rev. Lett. 101, 131801 (2008) arXiv:0804.4157 [astro-ph]].

[26] K. Nakayama, F. Takahashi and T. T. Yanagida, Phys. Lett. B 697, 275 (2011) arXiv:1010.5693 [hep-ph]].

[27] T. Kobayashi, F. Takahashi, T. Takahashi and M. Yamaguchi, JCAP 1203, 036 (2012) [arXiv:1111.1336 [astro-ph.CO]].

[28] K. S. Jeong and F. Takahashi, JHEP 1208 (2012) 017 [arXiv:1201.4816 [hep-ph]].

[29] K. Choi, K. -Y. Choi and C. S. Shin, arXiv:1208.2496 [hep-ph].

[30] M. Cicoli, J. P. Conlon and F. Quevedo, arXiv:1208.3562 [hep-ph].

[31] T. Higaki and F. Takahashi, arXiv:1208.3563 [hep-ph].

[32] K. Nakayama, F. Takahashi and T. T. Yanagida, Phys. Rev. D 84, 123523 (2011) arXiv:1109.2073 [hep-ph]].

[33] T. Higaki, K. Kamada and F. Takahashi, JHEP 1209, 043 (2012) arXiv:1207.2771 [hep-ph]]. 
[34] M. Grana, Phys. Rept. 423, 91 (2006) hep-th/0509003 ; M. R. Douglas and S. Kachru, Rev. Mod. Phys. 79, 733 (2007) hep-th/0610102.

[35] R. Blumenhagen, B. Kors, D. Lust and S. Stieberger, Phys. Rept. 445, 1 (2007) hep-th/0610327.

[36] K. Nakayama, F. Takahashi and T. T. Yanagida, arXiv:1209.2583 [hep-ph].

[37] T. Asaka, K. Hamaguchi, M. Kawasaki and T. Yanagida, Phys. Rev. D 61, 083512 (2000) hep-ph/9907559]; V. N. Senoguz and Q. Shafi, Phys. Lett. B 596, 8 (2004) hep-ph/0403294.

[38] K. Nakayama and F. Takahashi, JCAP 1110, 033 (2011) [arXiv:1108.0070 [hep-ph]]; JCAP 1205, 035 (2012) arXiv:1203.0323 [hep-ph]].

[39] E. J. Copeland, A. R. Liddle, D. H. Lyth, E. D. Stewart and D. Wands, Phys. Rev. D 49, 6410 (1994) astro-ph/9401011; G. R. Dvali, Q. Shafi and R. K. Schaefer, Phys. Rev. Lett. 73, 1886 (1994) hep-ph/9406319]; A. D. Linde and A. Riotto, Phys. Rev. D 56, 1841 (1997) hep-ph/9703209.

[40] G. Lazarides and C. Panagiotakopoulos, Phys. Rev. D 52, 559 (1995) hep-ph/9506325.

[41] M. Kawasaki, M. Yamaguchi and T. Yanagida, Phys. Rev. Lett. 85, 3572 (2000) hep-ph/0004243]; Phys. Rev. D 63, 103514 (2001) hep-ph/0011104.

[42] W. Buchmuller, L. Covi and D. Delepine, Phys. Lett. B 491, 183 (2000) hep-ph/0006168.

[43] K. Nakayama, F. Takahashi and T. T. Yanagida, JCAP 1012, 010 (2010) arXiv:1007.5152 [hep-ph]].

[44] M. Fujii and K. Hamaguchi, Phys. Lett. B 525, 143 (2002) hep-ph/0110072.

[45] N. Arkani-Hamed, S. Dimopoulos, G. F. Giudice and A. Romanino, Nucl. Phys. B 709, 3 (2005) hep-ph/0409232.

[46] K. S. Jeong, M. Shimosuka and M. Yamaguchi, JHEP 1209, 050 (2012) arXiv:1112.5293 [hep-ph]].

[47] M. Ibe and T. T. Yanagida, Phys. Lett. B 709, 374 (2012) [arXiv:1112.2462 [hep-ph]]. 
[48] W. Buchmuller, V. Domcke and K. Schmitz, Phys. Lett. B 713, 63 (2012) arXiv:1203.0285 [hep-ph]].

[49] J. Hisano, S. Matsumoto and M. M. Nojiri, Phys. Rev. Lett. 92, 031303 (2004) hep-ph/0307216]; J. Hisano, S. .Matsumoto, M. M. Nojiri and O. Saito, Phys. Rev. D 71, 063528 (2005) hep-ph/0412403.

[50] M. Fukugita, T. Yanagida, Phys. Lett. B174, 45 (1986).

[51] T. Yanagida, in Proceedings of the "Workshop on the Unified Theory and the Baryon Number in the Universe", Tsukuba, Japan, Feb. 13-14, 1979, edited by O. Sawada and A. Sugamoto, KEK report KEK-79-18, p. 95, and "Horizontal Symmetry And Masses Of Neutrinos" , Prog. Theor. Phys. 64 (1980) 1103; M. Gell-Mann, P. Ramond and R. Slansky, in "Supergravity" (North-Holland, Amsterdam, 1979) eds. D. Z. Freedom and P. van Nieuwenhuizen, Print-80-0576 (CERN); see also P. Minkowski, Phys. Lett. B 67, 421 (1977).

[52] For a review, see W. Buchmuller, P. Di Bari and M. Plumacher, Annals Phys. 315 (2005) 305 arXiv:hep-ph/0401240]; W. Buchmuller, R. D. Peccei, T. Yanagida, Ann. Rev. Nucl. Part. Sci. 55, 311-355 (2005). |hep-ph/0502169]; S. Davidson, E. Nardi and Y. Nir, Phys. Rept. 466, 105 (2008) [arXiv:0802.2962 [hep-ph]].

[53] K. S. Jeong and F. Takahashi, JHEP 1207, 170 (2012) [arXiv:1204.5453 [hep-ph]].

[54] L. Randall and R. Sundrum, Nucl. Phys. B 557, 79 (1999) hep-th/9810155; J. A. Bagger, T. Moroi and E. Poppitz, JHEP 0004, 009 (2000) hep-th/9911029.

[55] G. F. Giudice, M. A. Luty, H. Murayama and R. Rattazzi, JHEP 9812, 027 (1998) hep-ph/9810442.

[56] K. Choi and H. P. Nilles, JHEP 0704, 006 (2007) hep-ph/0702146 [hep-ph]].

[57] N. Okada, Phys. Rev. D 65, 115009 (2002) hep-ph/0202219.

[58] R. D. Peccei and H. R. Quinn, Phys. Rev. Lett. 38, 1440 (1977).

[59] For a review, see J. E. Kim, Phys. Rept. 150, 1 (1987); H. Y. Cheng, Phys. Rept. 158, 1 (1988).

[60] T. Gherghetta, G. F. Giudice and J. D. Wells, Nucl. Phys. B 559, 27 (1999) hep-ph/9904378. 
[61] J. L. Feng, T. Moroi, L. Randall, M. Strassler and S. -f. Su, Phys. Rev. Lett. 83, 1731 (1999) hep-ph/9904250.

[62] M. Ibe, S. Matsumoto and T. T. Yanagida, Phys. Rev. D 85, 095011 (2012) arXiv:1202.2253 [hep-ph]].

[63] J. M. Cline and S. Raby, Phys. Rev. D 43, 1781 (1991); R. J. Scherrer, J. M. Cline, S. Raby and D. Seckel, Phys. Rev. D 44, 3760 (1991).

[64] I. Affleck and M. Dine, Nucl. Phys. B 249, 361 (1985); M. Dine, L. Randall and S. D. Thomas, Nucl. Phys. B 458, 291 (1996) arXiv:hep-ph/9507453.

[65] M. Kawasaki, T. Moroi and T. Yanagida, Phys. Lett. B 383, 313 (1996) hep-ph/9510461.

[66] A. Arvanitaki, S. Dimopoulos, S. Dubovsky, N. Kaloper and J. March-Russell, Phys. Rev. D 81, 123530 (2010) [arXiv:0905.4720 [hep-th]].

[67] F. Takahashi and T. T. Yanagida, Phys. Lett. B 698, 408 (2011) arXiv:1101.0867 [hep-ph]]. 